\title{
Significant correlation between the acceleration of platelet aggregation and phosphorylation of HSP27 at Ser-78 in diabetic patients
}

\author{
HARUHIKO TOKUDA $^{1,2}$, KENJI KATO ${ }^{2}$, SENJI KASAHARA ${ }^{3}$, RIE MATSUSHIMA-NISHIWAKI ${ }^{2}$, \\ TAKAHIKO MIZUNO ${ }^{1}$, SEIKO SAKAKIBARA ${ }^{1}$ and OSAMU KOZAWA ${ }^{2}$ \\ ${ }^{1}$ Department of Clinical Laboratory, National Center for Geriatrics and Gerontology, Obu, Aichi 474-8511; \\ ${ }^{2}$ Department of Pharmacology and ${ }^{3}$ First Department of Internal Medicine, Gifu University, \\ Graduate School of Medicine, Gifu 501-1194, Japan
}

Received June 28, 2012; Accepted August 30, 2012

DOI: $10.3892 / \mathrm{ijmm} .2012 .1146$

\begin{abstract}
To clarify the mechanism underlying a high risk of thrombotic complications in diabetic patients, we investigated the relationship between HSP27 phosphorylation and the platelet activation induced by adenosine diphosphate (ADP) in diabetic patients. Platelet-rich plasma was prepared from the blood of type 2 diabetes mellitus (DM) patients. By measuring the dose response of platelet aggregation to ADP, an individual $\mathrm{ED}_{50}$ was determined. Based on the normal range identified in non-DM controls, the subjects were divided into a hyper-aggregate (Group 1) and a normo- or hypo-aggregate group (Group 2). The protein phosphorylation was analyzed by western blotting. The release of PDGF-AB and sCD40 ligand (sCD40L) was measured by ELISA. In both groups, ADP induced HSP27 phosphorylation at Ser-78 and Ser-82. The phosphorylation at Ser-78 and the release of both PDGF-AB and $\mathrm{SCD} 40 \mathrm{~L}$ induced by a low dose of ADP $(1 \mu \mathrm{M})$ in Group 1 were significantly higher than these values in Group 2. There was a significant relationship between the ADP-induced HSP27 phosphorylation level at Ser-78 and the ADP $\mathrm{ED}_{50}$ value of platelet aggregation. The ADP $(1 \mu \mathrm{M})$-induced phosphorylation of HSP at Ser-78 observed in the platelets from Group 1 was inhibited by PD98059 or SB203580. The use of aspirin ameliorated the accelerated microaggregation of platelets in Group 1, and the low-dose ADP-induced phosphorylation of HSP27 at Ser-78 was no longer observed. These results strongly suggest that the phosphorylation of HSP27 at Ser-78 is correlated with the
\end{abstract}

Correspondence to: Dr Haruhiko Tokuda, Department of Clinical Laboratory, National Center for Geriatrics and Gerontology, Obu, Aichi 474-8511, Japan

E-mail: tokuda@ncgg.go.jp

Key words: platelet aggregation, adenosine diphosphate, HSP27, phosphorylation, diabetes acceleration of platelet aggregation induced by ADP in type 2 DM patients.

\section{Introduction}

Platelets play a central role in thrombus formation, a pathological process associated with vascular diseases (1). Thrombosis is a critical event associated with myocardial infarction and stroke, major concerns of high mortality (2). Platelet activation is triggered by initial tethering at injured vascular sites, mediated by the formation of glycoprotein Ib/IX/V and von Willebrand factor complex. The release of adenosine diphosphate (ADP), thrombin, epinephrine and thromboxane A2. from activated platelets are rapidly generated to repair vascular injury (1). The release of inflammatory and mitogenic mediators including CD40 ligand (CD40L) and platelet-derived growth factor-AB (PDGF-AB) from platelets alters the vascular endothelial-cell function, resulting in atherosclerosis (1).

Type 2 diabetes mellitus (DM) is a major global health problem (3). These patients have an increased risk of vascular complications due to atherosclerosis such as cardiovascular disease (4), so the control of platelet aggregation is a clinical issue to improve the prognosis of DM patients. Although antiplatelet therapy, such as aspirin, is widely used in DM patients for the prevention of ischemic cardiovascular diseases (5), the existence of 'aspirin-resistance' is well known, but its mechanism remains to be clarified (6). Regarding platelet functions, we previously reported that irreversible platelet microaggregation could be induced by a low dose of ADP $(1 \mu \mathrm{M})$ in the majority of type 2 DM patients, and that the $\mathrm{P} 2 \mathrm{Y} 12$ receptor plays a key role in the hypersensitivity of platelet aggregation (7). We also reported that the collagen-induced activation of p44/p42 mitogen-activated protein (MAP) kinase and p38 MAP kinase are related to the platelet hyper-aggregation in type $2 \mathrm{DM}$ patients (8).

In response to biological stress, heat shock proteins (HSPs) are induced in both prokaryotic and eukaryotic cells (9). Among them, low-molecular-weight HSPs including HSP27 and $\alpha \mathrm{B}$ crystallin possess high homology in their amino acid 
sequences, $\alpha$-crystallin domain, and are known to function as molecular chaperones $(10,11)$. Human HSP27 is reportedly phosphorylated at three serine residues (Ser-15, Ser-78 and Ser-82) (12). HSP27 in a resting state exists in an aggregated form. Once phosphorylated, HSP27 is rapidly dissociated, resulting in the loss of its molecular chaperone activity $(13,14)$. We previously reported that the ADP-induced HSP27 phosphorylation by p44/p42 MAP kinase and p38 MAP kinase is correlated with the secretion of granules, such as PDGF-AB, from human platelets (15). However, the clinical relevance of HSP27 phosphorylation in platelets has not yet been clarified.

Herein, we investigated the relationship between HSP27 phosphorylation and the platelet activation induced by ADP in type $2 \mathrm{DM}$ patients. Our results strongly suggest that the phosphorylation of HSP27 is closely related to the acceleration of platelet aggregation induced by ADP in type $2 \mathrm{DM}$ patients.

\section{Materials and methods}

Materials. ADP was purchased from Sigma Chemical Co. (St. Louis, MO, USA). PD98059 and SB203580 were purchased from Calbiochem-Novabiochem Corporation (La Jolla, CA, USA). Anti-HSP27, anti-phospho-HSP27 (Ser-15) and anti-phospho-HSP27 (Ser-78) antibodies were purchased from Stressgen Biotechnologies (Victoria, BC, Canada). Anti-phospho-HSP27 (Ser-82) antibodies were purchased from BioMol Research Laboratories (Plymouth Meeting, PA, USA). The PDGF-AB enzyme-linked immunosorbent assay (ELISA) kit and sCD40L ELISA kit were purchased from R\&D Systems (Minneapolis, MN, USA). The other materials and chemicals were obtained from commercial sources.

Subjects. The inclusion criteria for the study were the presence of type $2 \mathrm{DM}$ according to the criteria of the World Health Organization. We excluded the patients who were being treated with non-steroidal anti-inflammatory drugs, statins, angiotensin-receptor blockers or angiotensin-converting enzyme inhibitors, which could affect their platelet functions. We also excluded the patients who were complicated with a malignancy, infectious diseases including hepatitis B and C, or autoimmune disorders. All participants were advised to avoid sleep deprivation or blood donation. The study was approved by the Committee of Ethics at the National Center for Geriatrics and Gerontology and Gifu University Graduate School of Medicine.

Blood sampling. Ten millilitres of blood was drawn from the vein between 8:00 and 9:00 after at least $15 \mathrm{~min}$ of bed rest to preserve steady state conditions. Sodium citrate $(14 \mu \mathrm{M})$ was added to the blood immediately as an anticoagulant, and platelet-rich plasma (PRP) was obtained by centrifugation at $155 \mathrm{x}$ g for $12 \mathrm{~min}$ at room temperature. Platelet-poor plasma (PPP) was prepared from the residual blood by centrifugation at $2,500 \mathrm{x} \mathrm{g}$ for $5 \mathrm{~min}$.

Platelet aggregation. Platelet aggregation was measured using an aggregometer (PA-200 apparatus; Kowa Co., Ltd.,
Tokyo, Japan) with a laser-scattering (LS) system as described previously $(7,15)$. In brief, PRP was preincubated at $37^{\circ} \mathrm{C}$ for $1 \mathrm{~min}$ with a stirring speed of $800 \mathrm{rpm}$. Platelet aggregation was monitored for $4 \mathrm{~min}$ after the addition of various doses of $\operatorname{ADP}(0,0.3,1$ and $3 \mu \mathrm{M})$. The percentage of transmittance of the isolated platelets was recorded as $0 \%$, and that of the appropriate PPP (blank) was recorded as $100 \%$. Platelet aggregation was then terminated by the addition of ice-cold EDTA $(10 \mathrm{mM})$. The conditioned mixture was collected and centrifuged at $10,000 \times \mathrm{g}$ at $4^{\circ} \mathrm{C}$ for $2 \mathrm{~min}$. The supernatant was collected and stored at $-80^{\circ} \mathrm{C}$. The pellet was washed twice with PBS and then lysed immediately by boiling in a lysis buffer containing $62.5 \mathrm{mM}$ Tris- $\mathrm{HCl}, \mathrm{pH} 6.8,2 \%$ sodium dodecyl sulfate (SDS), $50 \mathrm{mM}$ dithiothreitol and $10 \%$ glycerol for western blot analysis. When indicated, the PRP was pretreated with $20 \mu \mathrm{M}$ SB203580 or $50 \mu \mathrm{M}$ PD98059 for 15 min prior to the stimulation with ADP.

Determination of the individual $E D_{50}$ value of $A D P$. To evaluate individual platelet aggregation, we used an $\mathrm{ED}_{50}$ value for ADP-induced aggregation according to the assessment by an aggregometer with the LS system. The percentage of aggregation in each subject was analyzed at a dose of 0, 0.3, 1 and $3 \mu \mathrm{M}$ ADP. Using the ALOKA curve software included in the ALOKA RIA programs (ALOKA, Tokyo, Japan), a doseresponse curve was plotted. From the regression equation, the ADP dose corresponding to $50 \%$ aggregation was calculated and identified as the individual $\mathrm{ED}_{50}$ value for each subject.

Western blot analysis. Western blot analysis was performed as previously described (16). In brief, SDS-polyacrylamide gel electrophoresis (PAGE) was performed by the Laemmli method (17) in a $12.5 \%$ polyacrylamide gel. The proteins fractioned in the gels were transferred onto polyvinylidene fluoride (PVDF) membranes, and then the membranes were blocked with $5 \%$ fat-free dry milk in Tris-buffered saline with 0.1\% Tween-20 (TBS-T, $20 \mathrm{mM}$ Tris, pH 7.6, $137 \mathrm{mM} \mathrm{NaCl}$, $0.1 \%$ Tween-20) for $2 \mathrm{~h}$ before incubation with the indicated primary antibody. A peroxidase-labeled antibody raised in a goat against rabbit IgG (KPL, Gaithersburg, MD, USA) was used as the secondary antibody. The primary and secondary antibodies were diluted to their optimal concentrations with $5 \%$ fat-free dry milk in TBS-T. The peroxidase activity on the PVDF membrane was visualized with X-ray film by means of an ECL western blotting detection system (GE Healthcare, Buckinghamshire, UK) following the manufacturer's protocol. The bands were analyzed by densitometry using the ImageJ software program (National Institutes of Health, USA).

ELISA for sCD40 ligand (sCD4OL) or PDGF-AB. The levels of $\mathrm{SCD} 40 \mathrm{~L}$ and PDGF-AB in the supernatant of the conditioned mixture after platelet aggregation were determined using specific ELISA kits.

Statistical analysis. The statistical significance of the differences between two groups was assessed using an unpaired Student's t-test or the Chi square test. To analyze the correlation between two variables, the Spearman's rank-order correlation test was adopted. A probability of $>5 \%$ was considered to be statistically significant. 
Table I. Characteristics of the study subjects.

\begin{tabular}{|c|c|c|c|}
\hline & Group 1 & Group 2 & P-value \\
\hline Total number & 13 & 10 & \\
\hline Gender (F/M) & $6 / 7$ & $3 / 7$ & \\
\hline Age (years) & $68.1 \pm 7.7$ & $67.0 \pm 4.2$ & 0.695 \\
\hline DM duration (years) & $11.1 \pm 11.0$ & $5.2 \pm 5.5$ & 0.139 \\
\hline Height (cm) & $156.3 \pm 8.7$ & $163.3 \pm 7.9$ & 0.059 \\
\hline Weight (kg) & $58.0 \pm 9.8$ & $62.6 \pm 13.6$ & 0.349 \\
\hline BMI & $23.7 \pm 3.2$ & $23.3 \pm 3.8$ & 0.786 \\
\hline sBP (mmHg) & $121.8 \pm 16.9$ & $131.4 \pm 18.7$ & 0.213 \\
\hline $\mathrm{dBP}(\mathrm{mmHg})$ & $69.2 \pm 8.4$ & $78.2 \pm 10.2$ & $0.030^{\mathrm{a}}$ \\
\hline $\mathrm{HbA}_{1 \mathrm{c}}(\%)$ & $9.4 \pm 2.1$ & $7.8 \pm 1.1$ & $0.023^{\mathrm{a}}$ \\
\hline Glu (mg/dl) & $173.1 \pm 70.0$ & $131.9 \pm 26.4$ & 0.069 \\
\hline $\mathrm{TC}(\mathrm{mg} / \mathrm{dl})$ & $235.8 \pm 46.2$ & $218.4 \pm 34.7$ & 0.333 \\
\hline TG (mg/dl) & $120.1 \pm 36.9$ & $174.0 \pm 95.8$ & 0.120 \\
\hline HDL (mg/dl) & $54.5 \pm 11.5$ & $53.3 \pm 14.0$ & 0.818 \\
\hline Plt $\left(\mathrm{x} 10^{4}\right)$ & $22.3 \pm 6.1$ & $21.6 \pm 6.1$ & 0.766 \\
\hline $\mathrm{ADP}^{\mathrm{ED}}{ }_{50}(\mu \mathrm{M})$ & $0.706 \pm 0.2$ & $1.963 \pm 0.2$ & $<0.001^{\mathrm{b}}$ \\
\hline
\end{tabular}

DM patients $(\mathrm{n}=23)$ were divided into those in a hyper-aggregated state (Group 1; $\left.\mathrm{ED}_{50}<1.534 \mu \mathrm{M}\right)$ and those in a normo- or hypo-aggregated state (Group 2; $\mathrm{ED}_{50} \geq 1.534 \mu \mathrm{M}$ ) on the basis of the normal range calculated from the mean $\mathrm{ED}_{50}$ value of the non-DM control group (mean \pm 2 SEM range; $1.778 \pm 0.244 \mu \mathrm{M}$ ). F, female; $\mathrm{M}$, male; sBP, systolic blood pressure; dBP, diastolic blood pressure; HbA1c, hemoglobin A1c; Glu, plasma glucose; TC, total cholesterol; TG, triglyceride; HDL, high-density lipoprotein; Plt, platelets. The data are presented as the means $\pm \mathrm{SD}$. ${ }^{\mathrm{a}} \mathrm{P}<0.05,{ }^{\mathrm{b}} \mathrm{P}<0.01$.

\section{Results}

Characterization of the study groups. We first determined the standard range of the $\mathrm{ADP} \mathrm{ED}_{50}$ value for platelet aggregation analyzed by an aggregometer with the LS system in non-DM healthy control subjects $(n=52)$, and found that it was $1.778 \pm 0.244 \mu \mathrm{M}$ (mean $\pm 2 \mathrm{SEM})$. Based on the normal range identified in non-DM controls, the subjects were divided into two groups: Group 1, a hyper-aggregate group $\left(\mathrm{ED}_{50}<1.534 \mu \mathrm{M}\right)$ and Group 2, a normal or hypo-aggregate group $\left(\mathrm{ED}_{50} \geq 1.534 \mu \mathrm{M}\right)$. The clinical and biochemical characteristics of the subjects are presented in Table I. The HbA1c levels were significantly higher in Group 1 than in Group 2. However, the anthropometric indices were within the normal limits in both groups, and the differences in the metabolic variables were not significant between the two groups.

Platelet aggregation of the study groups. Representative patterns of ADP-induced platelet aggregation in the study groups analyzed by an aggregometer with the LS system are shown in Fig. 1A. ADP dose-dependently elicited platelet aggregation in both groups, however, irreversible formation of large aggregates induced by $1 \mu \mathrm{M}$ ADP was observed only in Group 1. In addition, as previously reported (7), spontaneous microaggregation without ADP stimulation was observed in several cases from Group 1.

Comparison of the ADP-induced HSP27 phosphorylation levels of platelets from type 2 DM patients in Groups 1 and 2. ADP has been reported to induce HSP27 phosphorylation in human platelets (18). We previously reported that
ADP-induced platelet granule secretion is correlated with the phosphorylation of HSP27 in healthy donors (15). It is well known that human HSP27 is phosphorylated at three serine residues (Ser-15, Ser-78 and Ser-82) (11). Thus, we first examined the effects of various doses of $\operatorname{ADP}(0.3,1$ and $3 \mu \mathrm{M})$ on the phosphorylation of HSP27 (Ser-15, Ser-78 and Ser-82) by western blot analysis. In both groups, ADP dose-dependently induced the phosphorylation of HSP27 at Ser-78 and Ser-82, without affecting the phosphorylation at Ser-15 (Fig. 1B). The ADP-induced levels of HSP27 phosphorylation (Ser-78) as quantified by western blotting using the ImageJ software program are shown in Fig. 2A. In Group 1, ADP $(1$ or $3 \mu \mathrm{M})$ caused a significant increase in HSP27 (Ser-78). On the other hand, only $3 \mu \mathrm{M}$ of ADP, but not $1 \mu \mathrm{M}$, increased the phosphorylation level of HSP27 (Ser-78) in Group 2 (Fig. 2A). The ADP-induced levels of HSP27 phosphorylation (Ser-82) are shown in Fig. 2B. Similarly, the same doses of ADP ( 1 or $3 \mu \mathrm{M}$ ) caused a significant increase in the phosphorylation of HSP27 (Ser-82) in Group 1, however, ADP increased the phosphorylation level of HSP27 (Ser-82) in Group 2 only at a dose of $3 \mu \mathrm{M}$ (Fig. 2B). The effect of ADP on the phosphorylation of HSP27 (Ser-78) was also more extensive than that on the phosphorylation of HSP27 (Ser-82).

Relationship between individual ADP ED 50 values and levels of HSP27 phosphorylation induced by ADP in type 2 $D M$ patients. To clarify whether there is a relationship between the platelet aggregation induced by ADP and the phosphorylation levels of HSP27 in platelets from type 2 DM patients, we plotted the levels of HSP27 phosphorylation (Ser-78 and Ser-82) induced by various doses of ADP against 
A
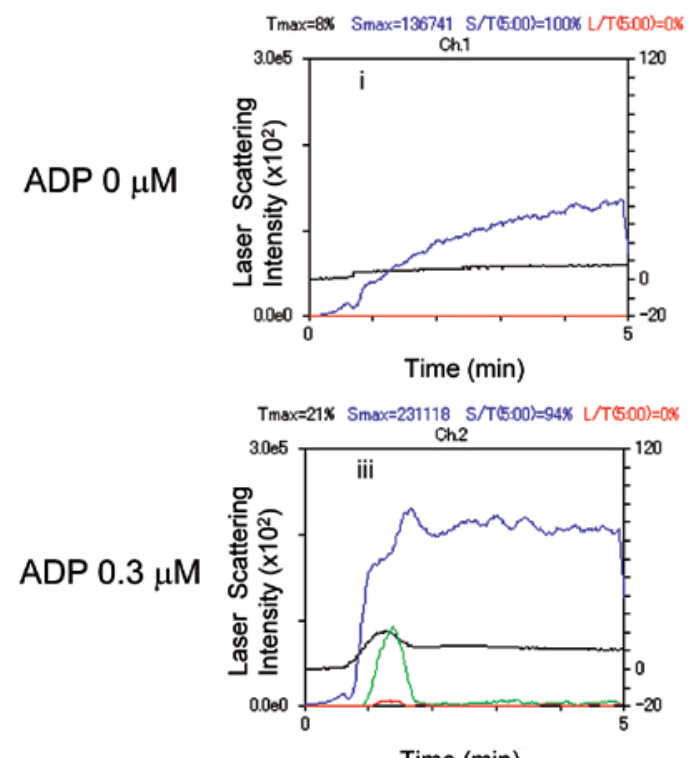

Time (min)
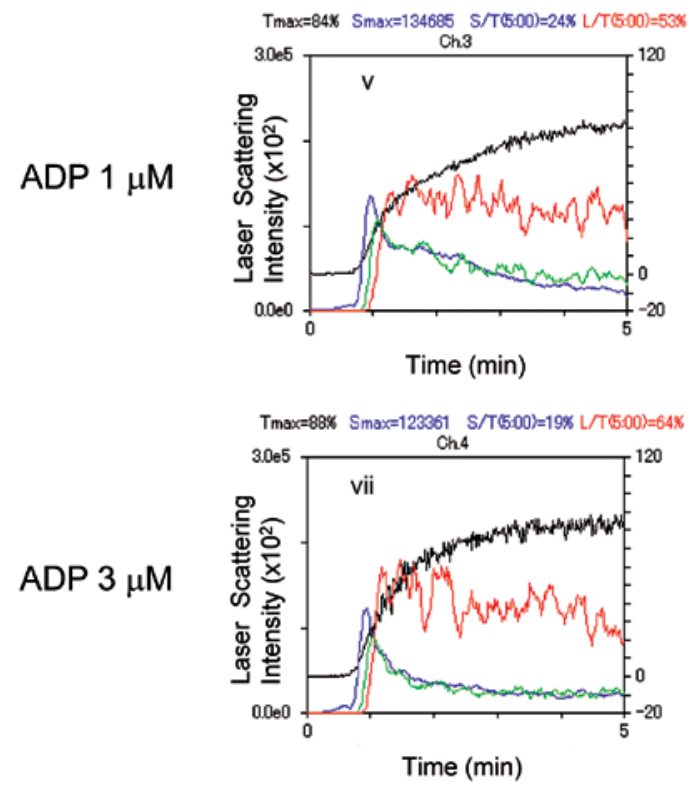

Group 2
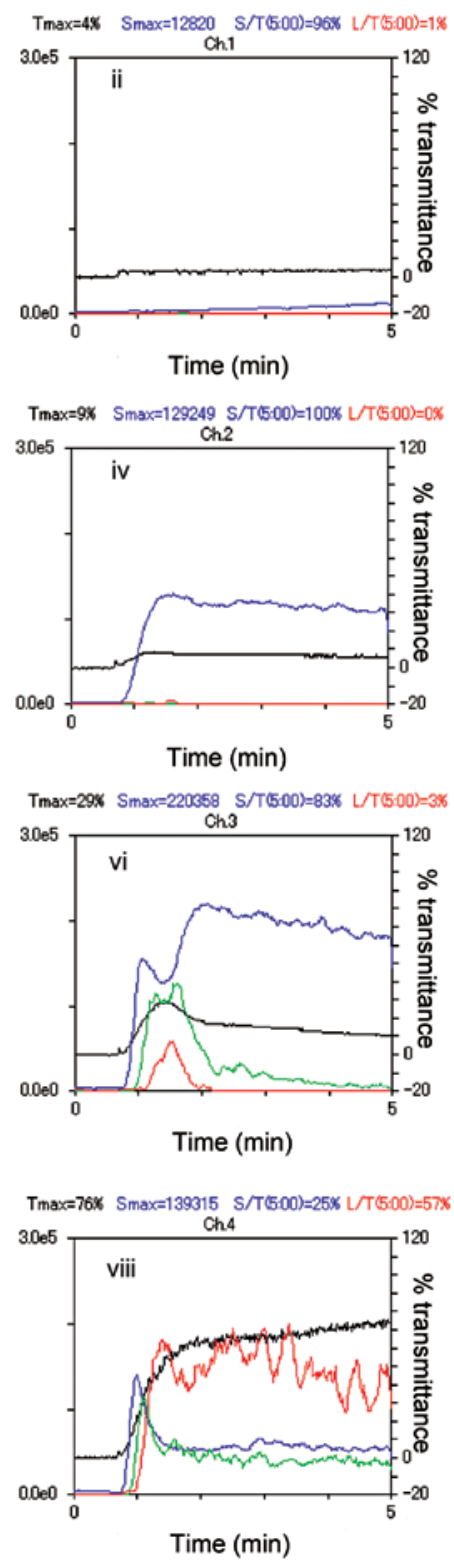
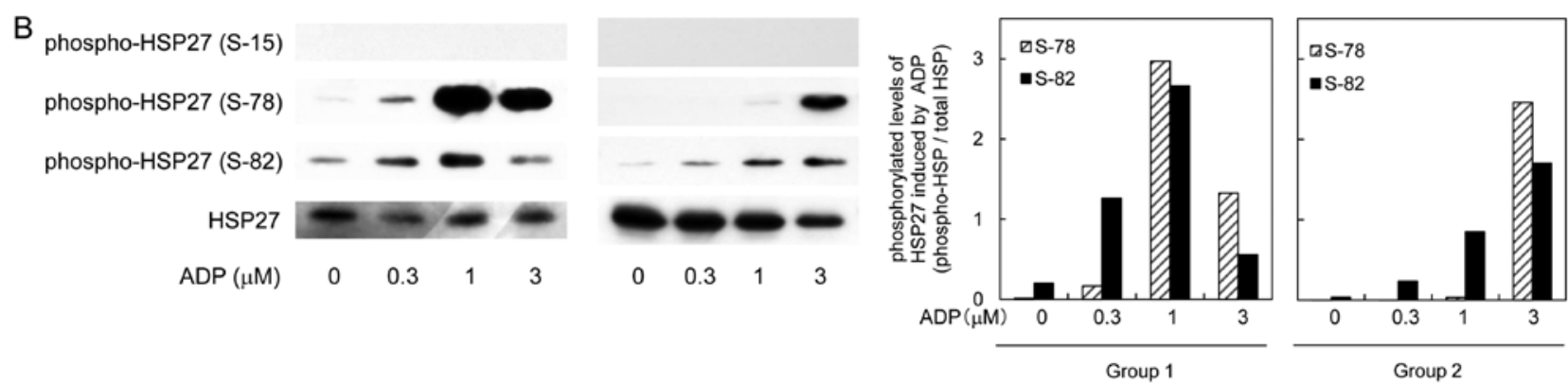

Figure 1. Representative patterns of platelet aggregation induced by various doses of ADP as detected by an aggregometer with the LS system and representative data showing the ADP-induced HSP27 phosphorylation in platelets from type 2 DM patients. PRP from type 2 DM patients was stimulated by various doses of $\operatorname{ADP}(0,0.3,1$ and $3 \mu \mathrm{M})$ in an aggregometer at $37^{\circ} \mathrm{C}$ for 4 min with a stirring speed of $800 \mathrm{rpm}$. (A) Time-dependent changes in the platelet aggregation after stimulation with 0 (i and ii), 0.3 (iii and iv), 1 (v and vi) and $3 \mu \mathrm{M}$ (vii and viii) are shown. The black line indicates the percentage of transmittance of each sample (the isolated platelets were recorded as $0 \%$, and platelet-free plasma was recorded as $100 \%)$. The blue line indicates small aggregates $(9-25 \mu \mathrm{m})$; green line, medium aggregates $(25-50 \mu \mathrm{m})$; red line, large aggregates $(50-70 \mu \mathrm{m})$. The distributions $(\%)$ of the aggregated particle size were measured with the LS methods. The DM patients were divided into groups based on a platelet aggregation-accelerated state (Group $\left.1, \mathrm{ED}_{50}<1.534 \mu \mathrm{M}\right)$ and a platelet aggregation-non-accelerated state (Group 2, $\mathrm{ED}_{50} \geq 1.534 \mu \mathrm{M}$ ) on the basis of the normal range calculated from the mean $\mathrm{ED}_{50}$ value of the non-DM control group (mean \pm 2 SEM range; $1.778 \pm 0.244 \mu \mathrm{M}$ ). (B) The reaction was terminated by the addition of an ice-cold EDTA (10 mM) solution. The extracts of platelets were subjected to western blot analysis using antibodies against total HSP27 and phospho-specific HSP27 (Ser-15, Ser-78 and Ser-82). The bands of phospho-HSP27 were quantified using the ImageJ software program and normalized to the total HSP27 band, and the ratio (phospho-HSP27/total HSP27) is presented for each value. Hatched bars indicate the phosphorylation ratio of HSP27 (Ser-78), and filled bars indicate the phosphorylation ratio of HSP27 (Ser-82). 
A

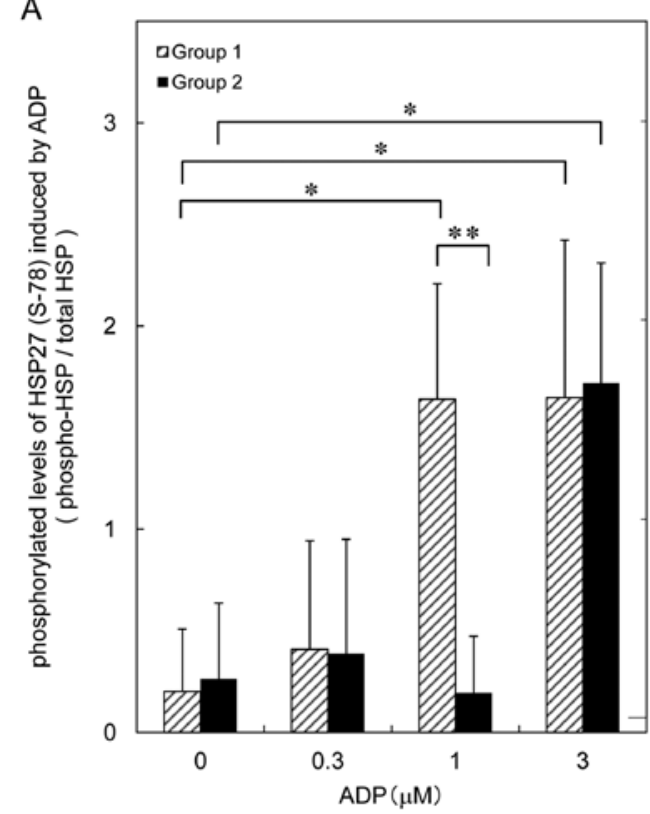

B

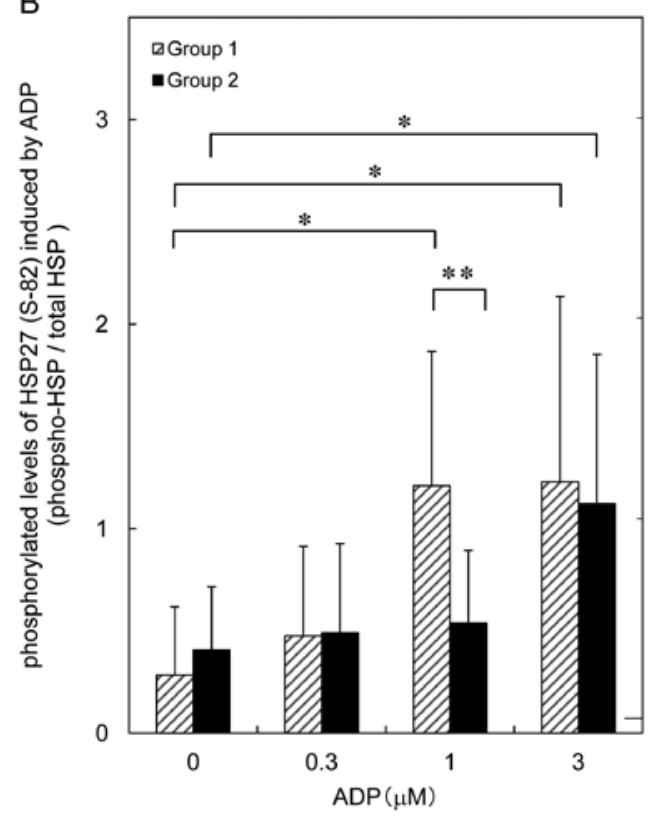

Figure 2. A comparison of the ADP-induced phosphorylation of HSP27 between Groups 1 and 2 by western blot analysis. DM subjects were divided into Group 1 (ED $\mathrm{ED}_{50}<1.534 \mu \mathrm{M}$, hatched bars, $\left.\mathrm{n}=13\right)$ and Group $2\left(\mathrm{ED}_{50} \geq 1.534 \mu \mathrm{M}\right.$, filled bars, $\left.\mathrm{n}=10\right)$ on the basis of the normal range calculated from the mean $\mathrm{ED}_{50}$ value of the non-DM control group (mean \pm 2 SEM range; $1.778 \pm 0.244 \mu \mathrm{M}$ ) and the levels of (A) phosphorylation of HSP27 (Ser-78) or (B) phosphorylation of HSP27 (Ser-82) were determined by western blot analysis. The bands of phospho-HSP27 were quantified using the ImageJ software program, and were normalized to the total HSP27 band and the ratio (phospho-HSP27/total HSP27) is presented. The values are presented as the means \pm SEM. ${ }^{*}<0.05$, for the value of Group 1 compared with that of Group 2 by Student's t-test.

the corresponding individual ADP ED 50 values (Fig. 3). For the phosphorylation of HSP27 at Ser-78, we observed a significant negative correlation for ADP at a concentration of $1 \mu \mathrm{M}$ (Fig. 3ii, $\mathrm{R}=0.836, \mathrm{P}<0.001, \mathrm{n}=23$ ). There was no significant relationship between the individual ADP $\mathrm{ED}_{50}$ values and the HSP27 (Ser-78) phosphorylation levels induced by 0.3 or $3 \mu \mathrm{M}$ of ADP (Fig. 3A-i and iii).

In regards to HSP27 (Ser-82) phosphorylation levels (Fig. 3B), there was a significant negative correlation with ADP at a concentration of $1 \mu \mathrm{M}$ (Fig. 3B-ii, $\mathrm{R}=0.474$, $\mathrm{P}=0.022, \mathrm{n}=23$ ). However, the correlation was weaker than that observed for the phosphorylation of HSP27 at Ser-78. No relationship was observed between the individual $\mathrm{ADP} \mathrm{ED}_{50}$ values and HSP27 (Ser-82) phosphorylation levels induced by 0.3 or $3 \mu \mathrm{M}$ of ADP (Fig. 3B-i and iii).

Effects of PD98059 or SB203580 on the phosphorylation of HSP27 (Ser-78) induced by $1 \mu M$ ADP in the platelets of type 2 DM patients classified into Group 1. We previously reported that PD98059, a specific inhibitor of MEK1/2 (19), or SB203580, a specific inhibitor of p38 MAP kinase (20), inhibits the phosphorylation of HSP27 induced by $3 \mu \mathrm{M}$ of ADP in the platelets from healthy donors and that this is related to granule secretion (15). We next examined the effects of PD98059 or SB203580 on the phosphorylation of HSP27 (Ser-78) induced by $1 \mu \mathrm{M}$ of ADP in the platelets of Group 1 type 2 DM patients. We previously confirmed that PD98059 $(50 \mu \mathrm{M})$ or SB203580 $(20 \mu \mathrm{M})$ potently suppresses the ADP-induced phosphorylation of $\mathrm{p} 44 / \mathrm{p} 42$ MAP kinase or that of p38 MAP kinase, respectively, in human platelets (15). In several cases from Group 1, PD98059 (50 $\mu \mathrm{M})$ significantly suppressed the phosphorylation of HSP27 (Ser-78) in the plate- lets induced by $1 \mu \mathrm{M}$ of ADP (Fig. 4). SB203580 also inhibited ADP (1 $\mu \mathrm{M})$-induced HSP27 (Ser-78) phosphorylation (Fig. 4).

Comparison of the release of $S C D 4 O L$ and the secretion of PDGF-AB induced by ADP from platelets of type 2 $D M$ patients in Groups 1 and 2. It has been reported that sCD40L, generated from CD40L, appears on the cell surface of activated platelets and is elevated in the plasma of type 2 DM patients (21). We therefore examined the effects of ADP on the release of $\mathrm{sCD} 40 \mathrm{~L}$ from the platelets of DM patients in Groups 1 and 2. The highest concentration of ADP $(3 \mu \mathrm{M})$ induced the release of sCD40L in both groups; however, a lower dose of ADP $(1 \mu \mathrm{M})$ caused the release in Group 1 but not in 2 (Fig. 5A).

We previously reported that the ADP-induced phosphorylation of HSP27 via p44/p42 MAP kinase and p38 MAP kinase is correlated with the secretion of PDGF-AB from the platelets of healthy donors (15). Therefore, we also compared the effects of ADP on the secretion of PDGF-AB from the platelets of Groups 1 and 2 . We observed that $3 \mu \mathrm{M}$ of ADP elicited PDGF-AB secretion from the platelets in both groups, but that the $1 \mu \mathrm{M}$ concentration of ADP significantly stimulated the release only in Group 1 (Fig. 5B).

Suppressive effect of aspirin on ADP-induced HSP27 phosphorylation (Ser-78) in Group 1 type 2 DM patients. Aspirin is widely used as an anti-platelet agent, and the adequate treatment with aspirin in DM patients for the prevention of cardiovascular diseases is recommended by the American Diabetes Association (5). We further examined the effects of aspirin therapy for 4 weeks on the phosphorylation of HSP27 (Ser-78) induced by ADP in several patients with type $2 \mathrm{DM}$ 

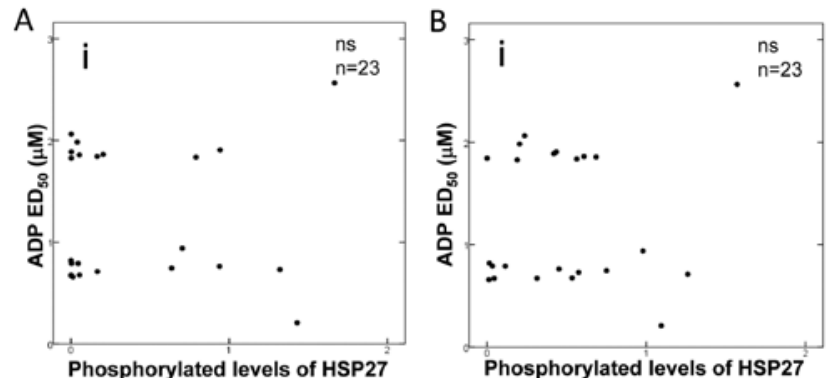

(S-78) induced by 0.3 H ADP

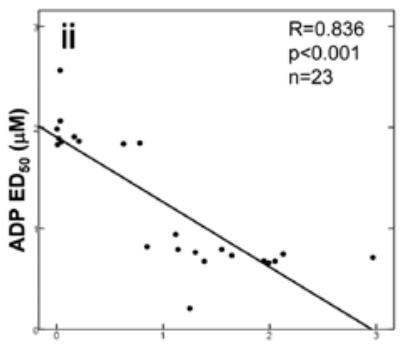

inglated levels of HSP27 (S-82) induced by $0.3 \mu \mathrm{M}$ ADP

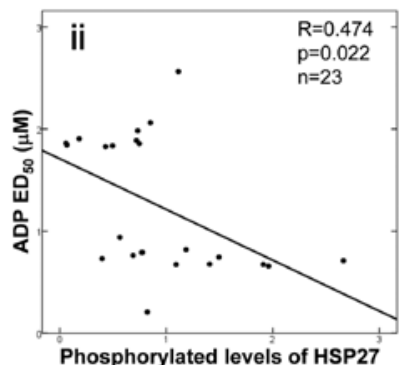

(S-78) ind

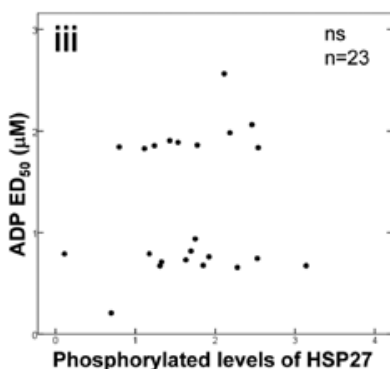

(S-78) induced by $3 \mu \mathrm{M}$ ADP

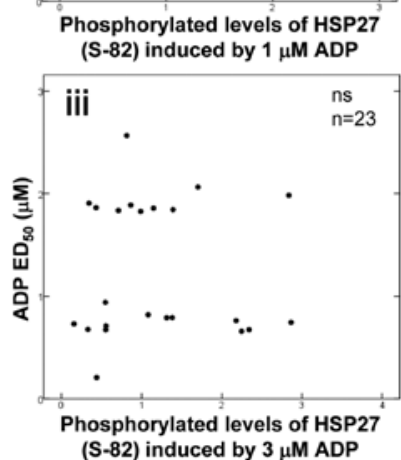

(S-82) induced by $3 \mu \mathrm{M}$ ADP

Figure 3. Relationship between individual $\mathrm{ADP} \mathrm{ED}_{50}$ values for the platelet aggregation and the levels of HSP27 phosphorylation (Ser-78 and Ser-82) in type $2 \mathrm{DM}$ patients. The individual $\mathrm{ADP} \mathrm{ED}_{50}$ values and the corresponding (A) phosphorylation of HSP27 (Ser-78) or (B) phosphorylation of HSP27 (Ser-82) ratio (phospho-HSP27/total HSP27) induced by various doses of ADP (panel i, $0.3 \mu \mathrm{M}$; panel ii, $1 \mu \mathrm{M}$; panel iii, $3 \mu \mathrm{M}$ ) were determined by western blot analysis using the ImageJ software program, and were plotted and analyzed by Spearman's rank-order correlation test. We observed a significant negative correlation of $\mathrm{ED}_{50}$ values with the phosphorylation of HSP27 (Ser78) (A) and the phosphorylation of HSP27 (Ser-82) (B), at a dose of $1 \mu \mathrm{M}$ of ADP (A-ii and B-ii).

who were classified into Group 1. The representative data are shown in Fig. 6. According to the results of the aggregometer, $1 \mu \mathrm{M}$ of ADP caused significant platelet aggregation in the case and almost $50 \%$ of the aggregates were of a large size $(50-70 \mu \mathrm{m})$ (Fig. 6A). Before aspirin therapy, the individual ADP $\mathrm{ED}_{50}$ value in the case was calculated to be $0.658 \mu \mathrm{M}$ (classified as Group 1). The phosphorylation of HSP27 (Ser78) was significantly induced by ADP even at $1 \mu \mathrm{M}$ in this patient (Fig. 6A). Treatment with an anti-platelet agent was proposed and the patient started to take aspirin at a dose of $100 \mathrm{mg}$ daily. After 4 weeks, the acceleration of ADP-induced platelet aggregation was significantly ameliorated. The platelet aggregates induced by $1 \mu \mathrm{M}$ of ADP were still observed, but $84 \%$ of them were microaggregates $(9-25 \mu \mathrm{m})$ (Fig. 6B). The individual ADP $\mathrm{ED}_{50}$ value was calculated to be $2.421 \mu \mathrm{M}$. In parallel with the amelioration of platelet aggregation, the phosphorylation of HSP27 (Ser-78) induced by ADP was markedly decreased and $1 \mu \mathrm{M}$ of ADP hardly elicited any HSP phosphorylation (Fig. 6B).

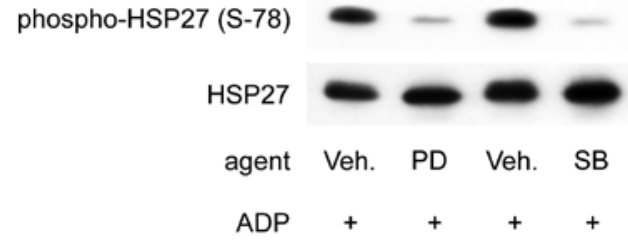

Figure 4. Effects of PD98059 or SB203580 on the ADP-induced phosphorylation of HSP27 (Ser-78) in the platelets from a type 2 DM patient classified into Group 1 (hyper-aggregated state). PD98059 (50 $\mu \mathrm{M})$, SB203580 $(20 \mu \mathrm{M})$, or vehicle were added to the platelet-rich plasma from the patient, then samples were incubated at $37^{\circ} \mathrm{C}$ for $15 \mathrm{~min}$, and then stimulated with $1 \mu \mathrm{M}$ of ADP for $4 \mathrm{~min}$ in an aggregometer. The reaction was terminated by the addition of an ice-cold EDTA $(10 \mathrm{mM})$ solution. The extracts of platelets were subjected to a western blot analysis using antibodies against total HSP27 and phosphospecific HSP27 (Ser-78). Representative results from three independent experiments are presented. Veh., vehicle; PD, PD98059; SB, SB203580.

\section{Discussion}

In the present study, we investigated the relationship between HSP27 phosphorylation and platelet aggregation induced by ADP in type $2 \mathrm{DM}$ patients. According to the individual $\mathrm{ED}_{50}$ value of ADP for platelet aggregation analyzed by the LS aggregometer, type $2 \mathrm{DM}$ patients were classified into two groups, hyper-aggregate group (Group 1) and normo- or hypoaggregate group (Group 2). We found that a low dose of ADP $(1 \mu \mathrm{M})$ caused significant phosphorylation of HSP27 (Ser-78 and Ser-82) in the patients with hyper-aggregation (Group 1), and that the effect of ADP on the phosphorylation of HSP27 (Ser-78) was more evident than that on HSP27 (Ser-82). We further investigated the relationship between the levels of HSP27 phosphorylation induced by various doses of ADP and the individual $\mathrm{ED}_{50}$ values for platelet aggregation in the study subjects, and showed that the phosphorylation of HSP27 only occurred when the platelets were stimulated by ADP $(1 \mu \mathrm{M})$, and that the extent of phosphorylation closely correlated with the individual $\mathrm{ED}_{50}$ values. Moreover, the relationship was more significant for the phosphorylation at Ser-78 than for that at Ser-82. These results strongly suggest that the phosphorylation of HSP27, especially at Ser-78, plays a role in the platelet hypersensitivity to ADP in type 2 DM patients. To the best of our knowledge, this is the first report clearly indicating the clinical and pathological significance of HSP27 phosphorylation in human platelets. Phosphorylated HSP27 is reportedly associated with the activation-dependent cytoskeleton in human platelets (18). The phosphorylation-mimicking mutants of HSP27 (Ser-15, Ser-78 and Ser-82) have been reported to lead to faster and stronger actin polymerization than the wild-type protein in human platelets (22). The conformational changes in the cytoskeleton and the actinpolymerization caused by HSP27 phosphorylation may be involved in the pathogenesis of platelet hyper-aggregation in type $2 \mathrm{DM}$ patients.

In addition, we previously reported that a low dose of ADP $(1 \mu \mathrm{M})$ induced microaggregation, and that this was significantly correlated with the HbAlc value, a clinical indicator of DM control, and that $\mathrm{P} 2 \mathrm{Y} 12$ receptors (not P2Y1) play a central role in the microaggregation (7). In the present study subjects, the individual HbAlc values were higher in 
A

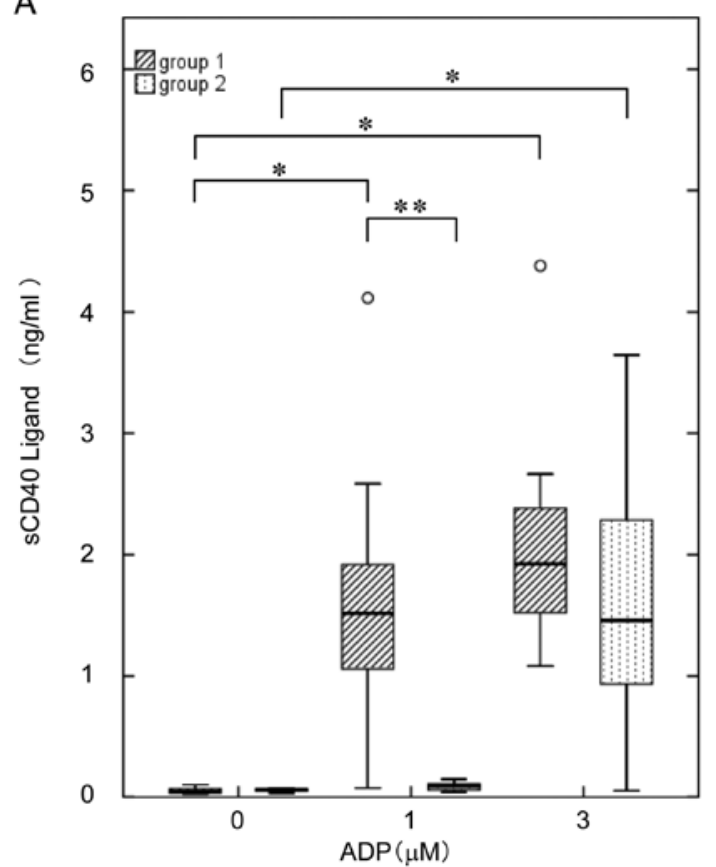

B

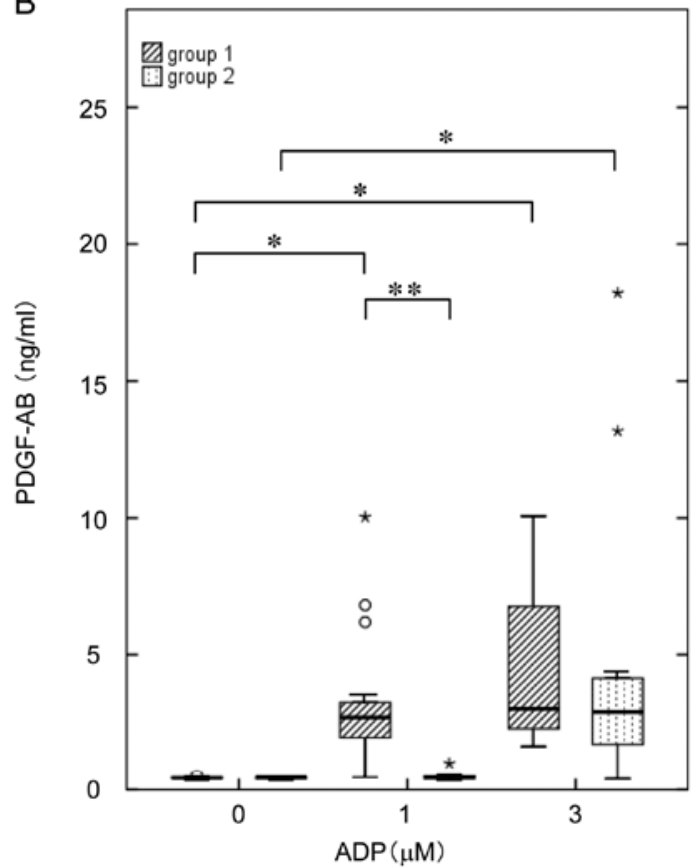

Figure 5. Comparison of the ADP-induced release of sCD40L and PDGF-AB between Groups 1 and 2 DM patients. The subjects were divided into Group 1 $\left(\mathrm{ED}_{50}<1.534 \mu \mathrm{M}\right.$, hatched bars, $\left.\mathrm{n}=13\right)$ and Group $2\left(\mathrm{ED}_{50} \geq 1.534 \mu \mathrm{M}\right.$, dotted bars, $\left.\mathrm{n}=10\right)$ on the basis of the normal range calculated from the mean $\mathrm{ED}_{50}$ value of the non-DM group (mean \pm 2 SEM range; $1.778 \pm 0.244 \mu \mathrm{M})$. The platelet-rich plasma was stimulated by various doses of ADP $(0,1$ and $3 \mu \mathrm{M})$ in an aggregometer at $37^{\circ} \mathrm{C}$ for $30 \mathrm{~min}$ with a stirring speed of $800 \mathrm{x} \mathrm{g}$, and the reaction was terminated by the addition of an ice-cold EDTA (10 mM) solution The level of (A) SCD40L and (B) PDGF-AB in the supernatant of the conditioned mixture after platelet aggregation was determined using specific ELISA kits. The thick horizontal lines correspond to the median values, the rectangles span the 25th to 75th percentiles, and the error bars indicate the range of the standard deviations. ${ }^{*} \mathrm{P}<0.05$, compared to the value without ADP, ${ }^{* *} \mathrm{P}<0.05$, for the value of Group 1 compared with that of Group 2 by Student's t-test.

the subjects of Group 1 than in Group 2, which was consistent with our previous findings. Based on our findings, it is probable that $\mathrm{P} 2 \mathrm{Y} 12$ receptor-mediated signaling is involved in the low-dose ADP $(1 \mu \mathrm{M})$-induced phosphorylation of HSP27 in the platelets derived from type 2 DM patients with a hyperaggregated status.

It has been previously reported that HSP27 phosphorylation is catalyzed by the MAP kinase superfamily $(11,23,24)$. In our present study, we also demonstrated that PD98059 (19) and SB203580 (20) both markedly inhibited the phosphorylation of HSP27 (Ser-78) induced by low-dose ADP $(1 \mu \mathrm{M})$ in Group 1 subjects, suggesting that the phosphorylation is dependent upon the activation of p44/p42 MAP kinase and p38 MAP kinase. Among the MAP kinase family members, we have found that only p44/p42 MAP kinase and p38 MAP kinase are involved in the phosphorylation of HSP27 induced by $3 \mu \mathrm{M}$ of ADP in human platelets from healthy donors, and that HSP27 phosphorylation is sufficient to induce granular secretion, but not platelet aggregation (15). In addition, we demonstrated that a low-dose of ADP $(1 \mu \mathrm{M})$ hardly elicits phosphorylation of HSP27 or the aggregation of platelets in healthy donors (15). In the present study, the relationship between the individual ADP $\mathrm{ED}_{50}$ value of platelet aggregation and the phosphorylated levels of HSP27 induced by ADP in the Group 1 DM patients was not significant at $3 \mu \mathrm{M}$, but was at $1 \mu \mathrm{M}$. Therefore, it seems likely that the involvement of HSP27 phosphorylation via $\mathrm{p} 44 / \mathrm{p} 42$ MAP kinase and p38 MAP kinase in platelet aggregation is limited to situations of pathological hyperaggregated status, such as type $2 \mathrm{DM}$.
We next examined the effect of ADP on the release of sCD40L and the secretion of PDGF-AB from the platelets and compared the results between Groups 1 and 2 . We observed that a low dose of ADP $(1 \mu \mathrm{M})$ significantly induced the release of both molecules in Group 1, but not in 2. This release induced by a low-dose of ADP seems to occur in parallel with the phosphorylation of HSP27. The CD40L released from platelets is known to be immediately cleaved into $\mathrm{SCD} 40 \mathrm{~L}$, which induces inflammatory responses in the endothelium, resulting in the production of reactive oxygen species, adhesion molecules, chemokines and tissue factors, major components of inflammatory responses promoting atherosclerosis (1). PDGF-AB is stored in the $\alpha$-granules of platelets and is released when the platelets are activated, after which it becomes involved in vascular atherosclerosis (25). Thus, it is likely that the low-dose ADP-induced phosphorylation of HSP27 plays significant roles in both the release of inflammatory or atherosclerogenic factors and the enhancement of platelet aggregation, resulting in an increased risk of vascular diseases in type $2 \mathrm{DM}$ patients.

In the present study, we examined the effect of aspirin on the phosphorylation of HSP27 (Ser-78) induced by ADP in several cases of type 2 DM classified into Group 1. We confirmed that 4 weeks of treatment with aspirin at a dose of $100 \mathrm{mg}$ daily caused significant improvement in the platelet hyper-aggregation, in parallel with the suppression of the lowdose ADP $(1 \mu \mathrm{M})$-stimulated HSP27 (Ser-78) phosphorylation levels in the platelets of these patients. Aspirin is a well known irreversible cyclooxygenase inhibitor which causes the 


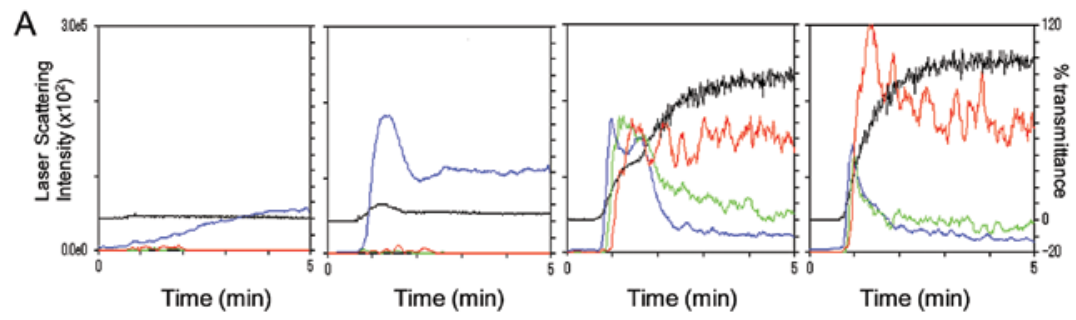

\begin{tabular}{lcccc} 
ADP $(\mu \mathrm{M})$ & 0 & 0.3 & 1 & 3 \\
Ratio $(\%)$ & & & & \\
\hline Small & 96 & 98 & 21 & 14 \\
Medium & 1 & 1 & 30 & 17 \\
Large & 3 & 1 & 49 & 70 \\
Total & 100 & 100 & 100 & 100 \\
\hline
\end{tabular}
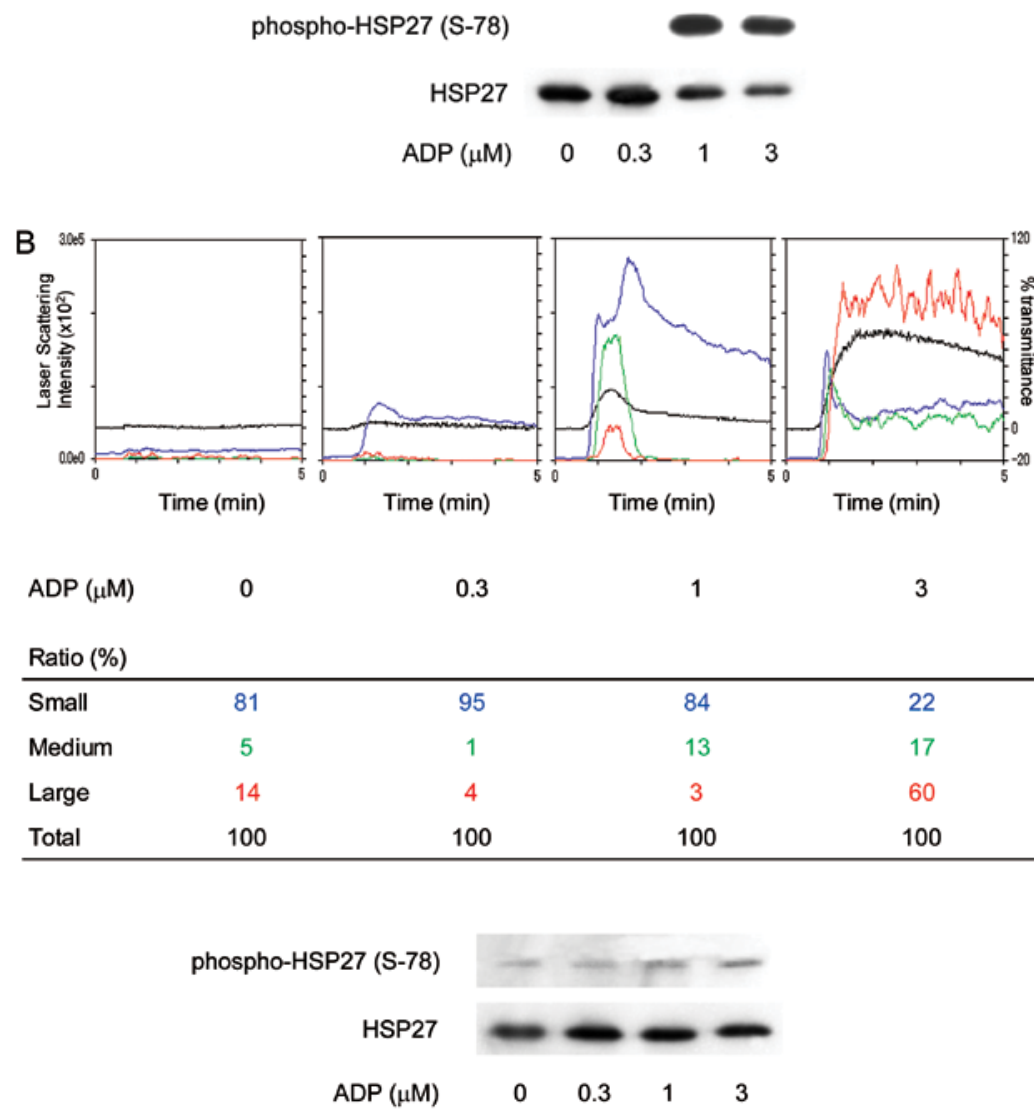

Figure 6. Representative data showing the effects of aspirin therapy on ADP-induced platelet aggregation and HSP27 phosphorylation (Ser-78) in Group 1 type 2 DM patients. The PRP was stimulated by various doses of ADP for $5 \mathrm{~min}$, and the reaction was terminated by the addition of an ice-cold EDTA $(10 \mathrm{mM})$ solution. The black line indicates the percentage of transmittance of each sample (the isolated platelets were recorded as $0 \%$, and the platelet-free plasma was recorded as $100 \%)$. The blue line indicates small aggregates $(9-25 \mu \mathrm{m})$; the green line, medium aggregates $(25-50 \mu \mathrm{m})$; the red line, large aggregates $(50-70 \mu \mathrm{m})$. The distributions $(\%)$ of the aggregated particle sizes were measured using a laser scattering method. (A) A representative result obtained from a type $2 \mathrm{DM}$ patient classified into Group 1 before aspirin medication, (B) a representative result from the same patient after aspirin therapy for 4 weeks.

inhibition of thromboxane A2 synthesis in human platelets (1), and its use is widely recommended for the prevention of cardiovascular diseases in type 2 DM patients (5). The significant effect of aspirin on the phosphorylation of HSP27 induced by low-dose ADP might indicate that the regulation of HSP27 phosphorylation is a novel therapeutic target for platelet hyper-aggregation in type $2 \mathrm{DM}$ patients. Moreover, the detection of HSP27 phosphorylation could be developed as a method for monitoring platelets for a hyper-aggregation status, particularly in the patients with vascular diseases associated with atherosclerosis. Further investigations would be required to clarify the detailed mechanisms underlying the functions of HSP27 in human platelets.

In conclusion, the phosphorylation of HSP27, particularly at Ser-78, is closely related to the acceleration of platelet microaggregation induced by ADP in type 2 DM patients. 


\section{Acknowledgements}

We are very grateful to Yoko Kawamura for her skillful technical assistance. This study was supported in part by the Grant-in-Aid for Scientific Research (20590565) from the Ministry of Education, Science, Sports and Culture of Japan and the Research Funding for Longevity Sciences (22-4) from National Center for Geriatrics and Gerontology (NCGG), Japan.

\section{References}

1. Davi G and Patrono C: Platelet activation and atherothrombosis. New Engl J Med 357: 2482-2494, 2007.

2. Furie B and Furie BC: Mechanism of thrombus formation. New Engl J Med 359: 938949, 2008.

3. Zimmet P, Albert KG and Shaw J: Global and social implications of the diabetes epidemic. Nature 414: 782-787, 2001.

4. Grundy SM, Benjamin IJ, Brurke GL, Chait A, Eckel RH, Howard BV, Mitch W, Smith SC, Jr and Sowers JR: Diabetes and cardiovasucular disease: a statement for healthcare professionals from the American Heart Association. Circulation 100: 1134-1146, 1999.

5. Colwell JA: American Diabetes Association. Aspirin therapy in diabetes. Diabetes Care 26 (Suppl 1): S87-S88, 2003.

6. Hankey GJ and Eikelboom JW: Aspirin resistance. Lancet 367: 606-617, 2006

7. Matsuno H, Tokuda H, Ishisaki A, Zhou Y, Kitajima Y and Kozawa O: P2Y12 receptors play a significant role in the development of platelet microaggregation in patients with diabetes. $J$ Clin Endocrinol Metab 90: 920-927, 2005.

8. Hanai Y, Adachi S, Yasuda I, Takai S, Matsushima-Nishiwaki R, Kato H, Enomoto Y, Akamatsu S, Sakakibara S, Ogura S, et al: Collagen-induced p38 MAP kinase activation is a biomarker of platelet hyper-aggregation in patients with diabetes mellitus. Life Sci 85: 386-394, 2009

9. Hendric JP and Hartl FU: Molecular chaperone functions of heat-shock proteins. Ann Rev Biochem 62: 349-384, 1993.

10. Inaguma Y, Goto S, Shinohara H, Hasegawa K, Ohshima K and Kato K: Physiological and pathological changes in levels of the two small stress proteins, HSP27 and $\alpha \mathrm{B}$ crystallin, in rat hindlimb muscles. J Biochem 114: 378-384, 1993.

11. Benjamin IJ and McMillan DR: Stress (heat shock) proteins: molecular chaperones in cardiovascular biology and disease. Circ Res 83: 117-132, 1998.

12. Landry J, Lambert H, Zhou M, Lavoie JN, Hickey E, Weber LA and Anderson CW: Human HSP27 is phosphorylated at serines 78 and 82 by heat shock and mitogen-activated kinases that recognize the same amino acid motif as S6 kinase II. J Biol Chem 267: 794-803, 1992.
13. Kato K, Hasegawa K, Goto S and Inaguma Y: Dissociation as a result of phosphorylation of an aggregated form or the small stress protein, hsp27. J Biol Chem 269: 11274-11278, 1994.

14. Rogalla T, Ehrnsperger M, Preville X, Kotlyarov A, Lutsch G, Ducasse C, Paul C, Wieske M, Arrigo AP, Buchner J and Gaestel M: Regulation of Hsp27 oligomerization, chaperone function, and protective activity against oxidative stress/ tumor necrosis factor $\alpha$ by phosphorylation. J Biol Chem 274: 18947-18956, 1999.

15. Kato H, Takai S, Matsushima-Nishiwaki R, Adachi S, Minamitani C, Otsuka T, Tokuda H, Akamatsu S, Doi T, Ogura S and Kozawa O: HSP27 phosphorylation is correlated with ADP-induced platelet granule secretion. Arch Biochem Biophys 475: 80-86, 2008.

16. Kato $\mathrm{K}$, Ito $\mathrm{H}$, Hasegawa $\mathrm{K}$, Inaguma $\mathrm{Y}$, Kozawa $\mathrm{O}$ and Asano $\mathrm{T}$ : Modulation of the stress-induced synthesis of hsp27 and $\alpha \mathrm{B}$ crystallin by cyclic AMP in C6 rat glioma cells. J Neurochem 66: 946-950, 1996.

17. Laemmli UK: Cleavage of structural proteins during the assembly of the head of bacteriophage T4. Nature 227: 680-685, 1970.

18. Zhu Y, O'Neill S, Saklatvala J, Tassi L and Mendelsohn ME: Phosphorylated HSP27 associates with the activation-dependent cytoskeleton in human platelets. Blood 84: 3715-3723, 1994.

19. Alessi DR, Cuenda A, Cohen P, Dudley DY and Saltiel AR: PD98059 is a specific inhititor of the activation of mitogenactivated protein kinase in vitro and in vivo. J Biol Chem 270: 27489-27494, 1995.

20. Cuenda A, Rouse J, Doza YN, Meier R, Cohen P, Gallagher TF, Young PR and Lee JC: SB203580 is a specific inhibitor of a MAP kinase homologue which is stimulated by cellular stresses and interleukin-1. FEBS Lett 364: 229-233, 1995.

21. Santilli F, Davi G, Consoli A, Cipollone F, Mezzetti A, Falco A, Tarborelli T, Devangelio E, Ciabattoni G, Basili S and Patrono C: Thromboxane-dependent CD40 ligand release in type 2 diabetes mellitus. J Am Coll Cardiol 47: 391-397, 2006.

22. Butt E, Immler D, Meyer HE, Kotlyarov A, Laass K and Gaestel M: Heat shock protein 27 is a substrate of cGMPdependent protein kinase in intact human platelets: phosphorylation-induced actin polymerization caused by HSP27 mutants. J Biol Chem 276: 7108-7113, 2001.

23. Kyriakis JM and Avruch J: Sounding the alarm: protein kinase cascades activated by stress and inflammation. J Biol Chem 271: 24313-24316, 1996.

24. Guay J, Lambert H, Gingras-Breton G, Lavoie JN, Huot J and Landry J: Regulation of actin filament dynamics by p38 map kinase-mediated phosphorylation of heat shock protein 27. J Cell Sci 110: 357-368, 1997.

25. Rendu F and Brohard-Bohn B: The platelet release reaction: granules' constituents, secretion and functions. Platelets 12 : 261-273, 2001. 